\title{
Cloud Computing: An Internet Based Computing
}

\author{
Hardeep Singh ${ }^{1}$ \\ Student
}

\begin{abstract}
Present paper focuses on the overview of Cloud Computing. Cloud Computing is an Internet-based computing; where resources, software and information are provided to computers on-demand, like a public utility; is emerging as a platform for sharing resources like infrastructure, software and various applications. This paper does a literature survey on some of the prominent applications of Cloud Computing, and how they meet the requirements of reliability, availability of data, scalability of software and hardware systems and overall customer satisfaction.
\end{abstract}

\section{INTRODUCTION}

Imagine yourself in the world where the users of the computer of today's internet world don't have to run, install or store their application or data on their own computers, imagine the world where every piece of your information or data would reside on the Cloud (Internet). The underlying concept dates back to 1960 when John McCarthy opined that "computation may someday be organized as a public utility"; indeed it shares characteristics with service bureaus which date back to the 1960s. The term cloud had already come into commercial use in the early 1990s to refer to large ATM networks. By the turn of the 21st century, the term "cloud computing" had started to appear, although most of the focus at this time was on Software as a service (SaaS).

In 1999, Salesforce.com was established by Marc Benioff, Parker Harris, and his fellows. They applied many technologies of consumer web sites like Google and Yahoo! to business applications. They also provided the concept of "On demand" and "SaaS" with their real business and successful customers. The key for SaaS is being customizable by customer alone or with a small amount of help. Flexibility and speed for application development have been drastically welcomed and accepted by business users.

IBM extended these concepts in 2001, as detailed in the Autonomic Computing Manifesto -- which described advanced automation techniques such as self-monitoring, self-healing, self-configuring, and self-optimizing in the management of complex IT systems with heterogeneous storage, servers, applications, networks, security mechanisms, and other system elements that can be virtualized across an enterprise.

Amazon.com played a key role in the development of cloud computing by modernizing their data centers after the dot-com bubble and, having found that the new cloud architecture resulted in significant internal efficiency improvements, providing access to their systems by way of Amazon Web Services in 2005 on a utility computing basis.
2007 saw increased activity, with Google, IBM, and a number of universities embarking on a large scale cloud computing research project, around the time the term started gaining popularity in the mainstream press. It was a hot topic by mid-2008 and numerous cloud computing events had been scheduled.

In August 2008, Gartner Research observed that "organizations are switching from company-owned hardware and software assets to per-use service-based models" and that the "projected shift to cloud computing will result in dramatic growth in IT products in some areas and in significant reductions in other areas."

As a metaphor for the Internet, "the cloud" is a familiar cliché, but when combined with "computing", the meaning gets bigger and fuzzier. Some analysts and vendors define cloud computing narrowly as an updated version of utility computing: basically virtual servers available over the Internet. Others go very broad, arguing anything you consume outside the firewall is "in the cloud", including conventional outsourcing.

Cloud computing comes into focus only when you think about what we always need: a way to increase capacity or add capabilities on the fly without investing in new infrastructure, training new personnel, or licensing new software. Cloud computing encompasses any subscription-based or pay-per-use service that, in real time over the Internet, extends ICT's existing capabilities.

Cloud computing is at an early stage, with a motley crew of providers large and small delivering a slew of cloud-based services, from full-blown applications to storage services to spam filtering. Yes, utility-style infrastructure providers are part of the mix, but so are SaaS (software as a service) providers such as Salesforce.com. Today, for the most part, IT must plug into cloud-based services individually, but cloud computing aggregators and integrators are already emerging.

\section{CLOUD COMPUTING: THE CONCEPT}

Cloud computing is Internet ("cloud") based development and use of computer technology ("computing"). It is a style of computing in which dynamically scalable and often virtualized resources are provided as a service over the Internet. Users need not have knowledge of, expertise in, or control over the technology infrastructure "in the cloud" that supports them

The concept Incorporates Infrastructure as a Service (IaaS), Platform as a Service (PaaS) and Software as a Service (SaaS) as well as Web 2.0 and other recent technology trends which have the common theme of reliance on the Internet for satisfying the computing needs of the users. Examples of SaaS vendors include 
Salesforce.com and Google Apps, which provide common business applications online that are accessed from a web browser, while the software and data are stored on the servers.

The term cloud is used as a metaphor for the Internet, based on how the Internet is depicted in computer network diagrams, and is an abstraction for the complex infrastructure it conceals.

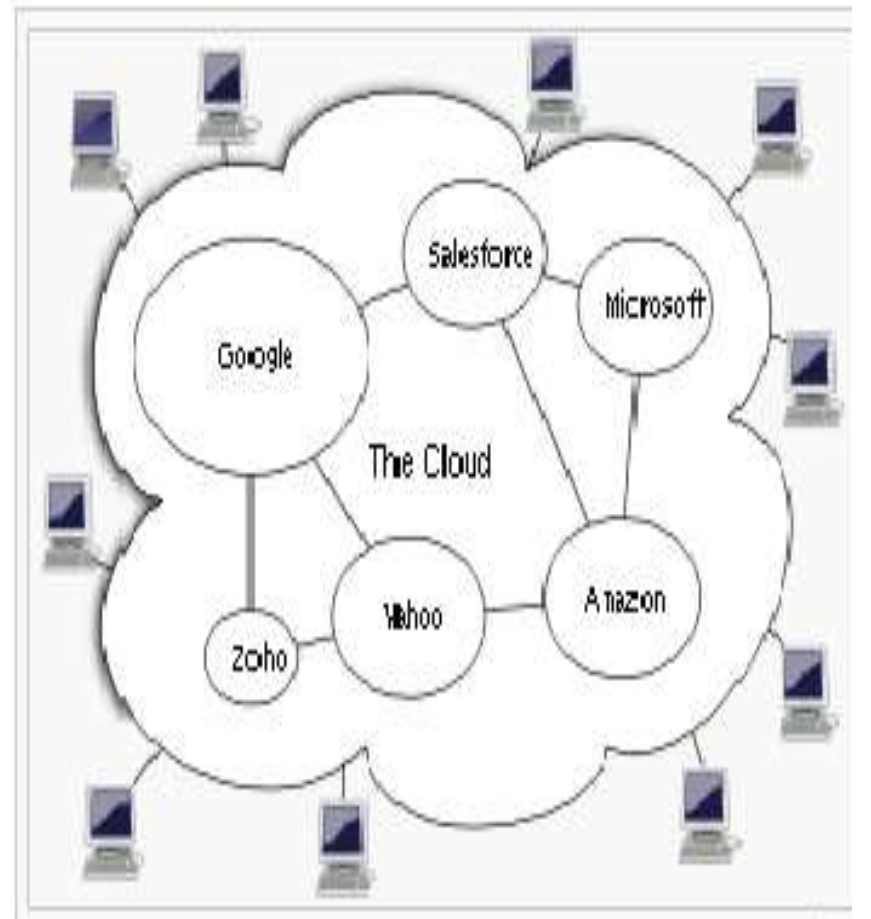

Cloud computing overview

\section{Figure 1 :Cloud Computing Overview}

\section{A. COMPARISON}

Cloud computing is often confused with grid computing ("a form of distributed computing whereby a 'super and virtual computer' is composed of a cluster of networked, loosely-coupled computers, acting in concert to perform very large tasks"), utility computing (the "packaging of computing resources, such as computation and storage, as a metered service similar to a traditional public utility such as electricity") and autonomic computing ("computer systems capable of self-management").

Indeed many cloud computing deployments as of 2009[update] depend on grids, have autonomic characteristics and bill like utilities - but cloud computing can be seen as a natural next step from the grid-utility model. Some successful cloud architectures have little or no centralized infrastructure or billing systems whatsoever, including peer-to-peer networks like Bit Torrent and Skype.

\section{B. IMPLEMENTATION}

The majority of cloud computing infrastructure as of 2009[update] consists of reliable services delivered through data centers and built on servers with different levels of virtualization technologies. The services are accessible anywhere that has access to networking infrastructure. The Cloud appears as a single point of access for all the computing needs of consumers. Commercial offerings need to meet the quality of service requirements of customers and typically offer service level agreements. Open standards are critical to the growth of cloud computing and open source software has provided the foundation for many cloud computing implementations.

\section{CHARACTERISTICS}

As customers generally do not own the infrastructure, they merely access or rent, they can avoid capital expenditure and consume resources as a service, paying instead for what they use. Many cloud-computing offerings have adopted the utility computing model, which is analogous to how traditional utilities like electricity are consumed, while others are billed on a subscription basis. Sharing "perishable and intangible" computing power among multiple tenants can improve utilization rates, as servers are not left idle, which can reduce costs significantly while increasing the speed of application development. A side effect of this approach is that "computer capacity rises dramatically" as customers do not have to engineer for peak loads. Adoption has been enabled by "increased high-speed bandwidth" which makes it possible to receive the same response times from centralized infrastructure at other sites.

\section{ECONOMICS}

Cloud computing users can avoid capital expenditure (CapEx) on hardware, software and services, rather paying a provider only for what they use. Consumption is billed on a utility (e.g. resources consumed, like electricity) or subscription (e.g. time based, like a newspaper) basis with little or no upfront cost. Other benefits of this time sharing style approach are low barriers to entry, shared infrastructure and costs, low management overhead and immediate access to a broad range of applications. Users can generally terminate the contract at any time (thereby avoiding return on investment risk and uncertainty) and the services are often covered by service level agreements with financial penalties.

According to Nicholas Carr the strategic importance of information technology is diminishing as it becomes standardized and cheaper. He argues that the cloud computing paradigm shift is similar to the displacement of electricity generators by electricity grids early in the 20th century.

\section{E. COMPANIES}

Providers including Amazon, Microsoft, Google, Sun and Yahoo exemplify the use of cloud computing. It is being adopted by individual users through large enterprises including General Electric, L'Oréal, and Procter \& Gamble. 


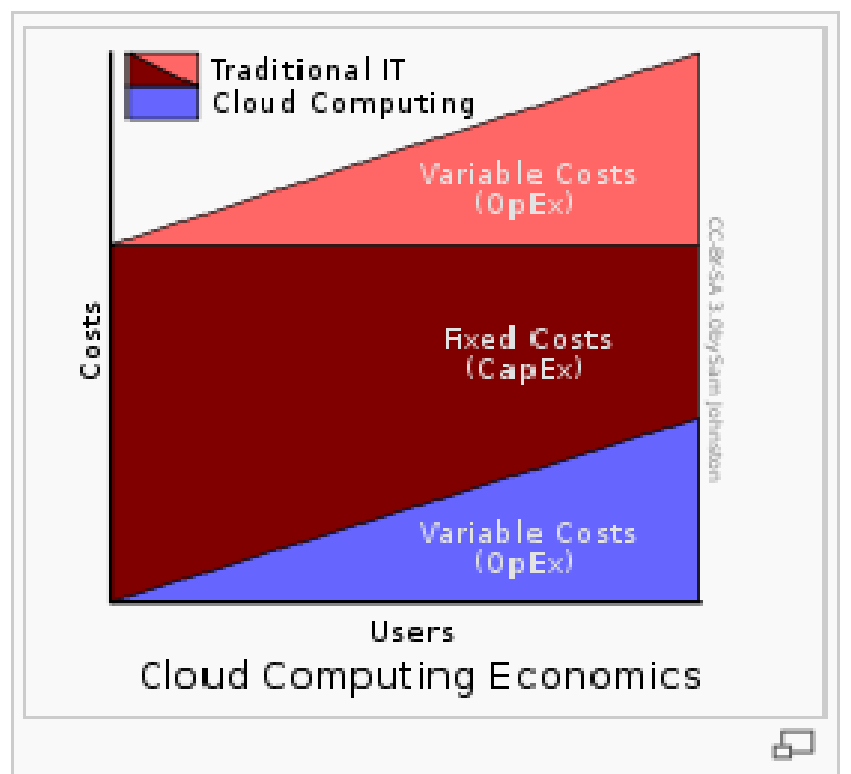

Figure 2 : Cloud Computing Economics

\section{CLOUD COMPUTING COMPONENTS}

\section{A. APPLICATION}

A cloud application leverages the Cloud in software architecture, often eliminating the need to install and run the application on the customer's own computer, thus alleviating the burden of software maintenance, ongoing operation, and support. For example:

Peer-to-peer / volunteer computing (Bittorrent, BOINC Projects, Skype)

Web application (Facebook)

Software as a service (Google Apps, SAP and Salesforce) Software plus services (Microsoft Online Services)

\section{B. CLIENT}

A cloud client consists of computer hardware and/or computer software which relies on cloud computing for application delivery, or which is specifically designed for delivery of cloud services and which, in either case, is essentially useless without it. For example:

Mobile (Android, iPhone, Windows Mobile)

Thin client (CherryPal, Zonbu, gOS-based systems)

Thick client / Web browser (Google Chrome, Mozilla Firefox)

\section{INFRASTRUCTURE}

Cloud infrastructure, such as Infrastructure as a service, is the delivery of computer infrastructure, typically a platform virtualization environment, as a service. For example:

Full virtualization (GoGrid, Skytap)

Management (RightScale)

Compute (Amazon Elastic Compute Cloud)

Platform (Force.com)

\section{PLATFORM}

A cloud platform, such as Platform as a service, the delivery of a computing platform, and/or solution stack as a service, facilitates deployment of applications without the cost and complexity of buying and managing the underlying hardware and software layers. For example:

Web application frameworks

Python Django (Google App Engine)

Ruby on Rails (Heroku)

.NET (Azure Services Platform)

Web hosting (Mosso)

Proprietary (Force.com)

\section{E. SERVICE}

A cloud service includes "products, services and solutions that are delivered and consumed in real-time over the Internet". For example, Web Services ("software system[s] designed to support interoperable machine-tomachine interaction over a network") which may be accessed by other cloud computing components, software, e.g., Software plus service, or end users directly. Specific examples include:

Identity (OAuth, OpenID)

Integration (Amazon Simple Queue Service)

Payments (Amazon Flexible Payments Service, Google Checkout, PayPal)

Mapping (Google Maps, Yahoo! Maps)

Search (Alexa, Google Custom Search, Yahoo! BOSS)

Others (Amazon Mechanical Turk)

\section{F. STORAGE}

Cloud storage involves the delivery of data storage as a service, including database-like services, often billed on a utility computing basis, e.g., per gigabyte per month. For example:

Database (Amazon SimpleDB, Google App Engine's BigTable datastore)

Network attached storage (MobileMe iDisk, Nirvanix CloudNAS)

Synchronization (Live Mesh Live Desktop component, MobileMe push functions)

Web service (Amazon Simple Storage Service, Nirvanix SDN)

\begin{tabular}{|c|}
\hline Clients \\
\hline Services \\
\hline Application \\
\hline Platform \\
\hline Storage \\
\hline Infrastructure \\
\hline
\end{tabular}

Figure. 3 : Cloud Computing Components

\begin{tabular}{|l|l|}
\hline Applications & $\begin{array}{l}\text { Facebook · Google Apps · SalesForce } \\
\text { Microsoft Online }\end{array}$ \\
\hline Client & Browser(Chrome) $\cdot$ Firefox C Cloud · \\
\hline
\end{tabular}




\begin{tabular}{|c|c|}
\hline & $\begin{array}{lll}\text { Mobile (Android · } & \text { iPhone) } & \text { Netbook } \\
\text { (EeePC } \text { MSI } & \text { Wind) } & \text { Nettop } \\
\text { (CherryPal } \cdot \text { Zonbu) } & & \end{array}$ \\
\hline Infrastructure & $\begin{array}{l}\text { BitTorrent } \cdot \text { EC2 } \cdot \text { GoGrid } \cdot \text { Sun Grid } \cdot \\
\text { 3tera }\end{array}$ \\
\hline Platforms & 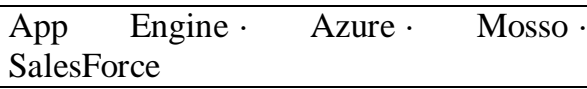 \\
\hline Services & Alexa $\cdot$ FPS $\cdot$ MTurk $\cdot$ SQS \\
\hline Storage & S3 - SimpleDB · SQL Services \\
\hline Standards & Ajax $\cdot$ Atom $\cdot$ HTML $5 \cdot$ REST \\
\hline
\end{tabular}

\section{A. PUBLIC CLOUD}

Public cloud or external cloud describes cloud computing in the traditional mainstream sense, whereby resources are dynamically provisioned on a fine-grained, selfservice basis over the Internet, via web applications/web services, from an off-site third-party provider who shares resources and bills on a fine-grained utility computing basis.

\section{B. PRIVATE CLOUD}

Private cloud and internal cloud are neologisms that some vendors have recently used to describe offerings that emulate cloud computing on private networks. These products claim to "deliver some benefits of cloud computing without the pitfalls", capitalizing on data security, corporate governance, and reliability concerns.

While an analyst predicted in 2008 that private

\section{ARCHITECTURE}

Cloud architecture, the systems architecture of the software systems involved in the delivery of cloud computing, comprises hardware and software designed by a cloud architect who typically works for a cloud integrator. It typically involves multiple cloud components communicating with each other over application programming interfaces, usually web services.

This closely resembles the UNIX philosophy of having multiple programs doing one thing well and working together over universal interfaces. Complexity is controlled and the resulting systems are more manageable than their monolithic counterparts.

Cloud architecture extends to the client, where web browsers and/or software applications access cloud applications.

Cloud storage architecture is loosely coupled, where metadata operations are centralized enabling the data nodes to scale into the hundreds, each independently delivering data to applications or user.

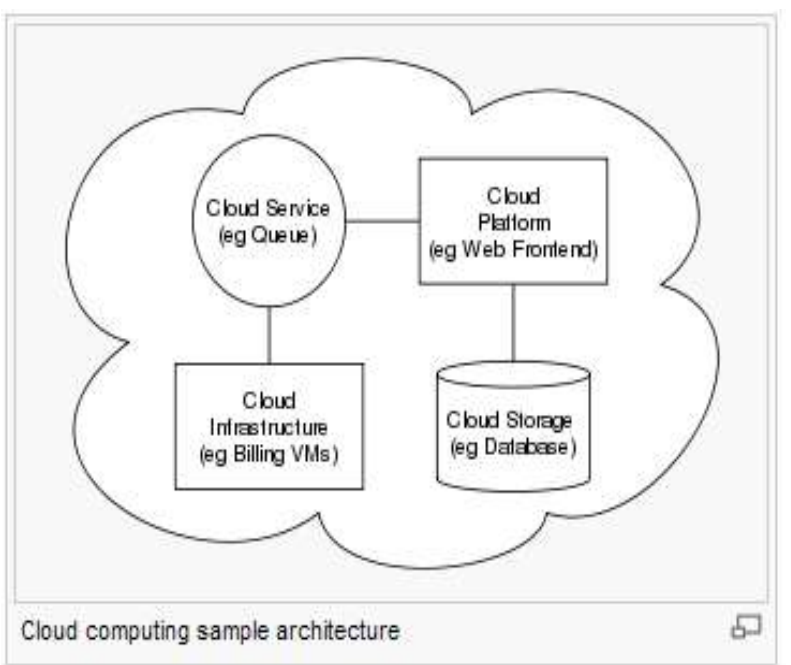

Figure 4 : Cloud Computing Architecture

\section{CLOUD COMPUTING TYPES} cloud networks would be the future of corporate IT, there is some uncertainty whether they are a reality even within the same firm. Analysts also claim that within five years a "huge percentage" of small and medium enterprises will get most of their computing resources from external cloud computing providers as they "will not have economies of scale to make it worth staying in the IT business" or be able to afford private clouds

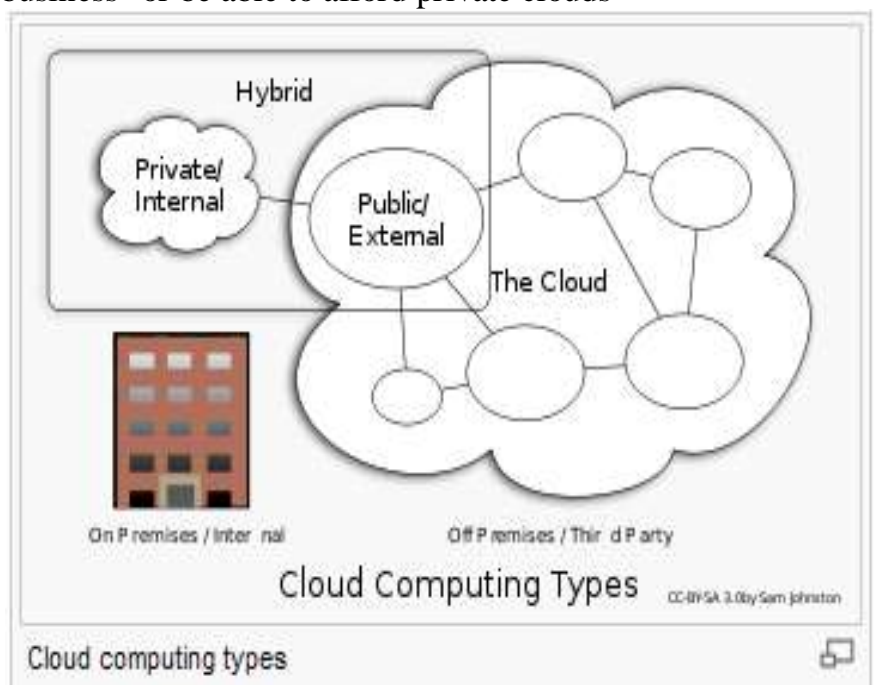

Figure 5 : Cloud Computing Architecture

$$
\text { V. ROLES }
$$

\section{A. PROVIDER}

A cloud computing provider or cloud computing service provider owns and operates live cloud computing systems to deliver service to third parties. The barrier to entry is also significantly higher with capital expenditure required and billing and management creates some overhead. Nonetheless, significant operational efficiency and agility advantages can be realized, even by small organizations, and server consolidation and virtualization rollouts are already well underway. Amazon.com was the first such provider, modernizing its data centers which, like most computer networks, were using as little as $10 \%$ of its capacity at any one time just to leave room for occasional spikes. This allowed small, fast-moving groups to add 
new features faster and easier, and they went on to open it up to outsiders as Amazon Web Services in 2002 on a utility computing basis.

\section{B. USER}

A user is a consumer of cloud computing. The privacy of users in cloud computing has become of increasing concern. The rights of users are also an issue, which is being addressed via a community effort to create a bill of rights.

\section{VENDOR}

A vendor sells products and services that facilitate the delivery, adoption and use of cloud computing. For example:

Computer hardware (Dell, HP, IBM, Sun Microsystems)

Storage (Sun Microsystems, EMC, IBM)

Infrastructure (Cisco Systems)

Computer software (3tera, Hadoop, IBM, RightScale)

Operating systems (Solaris, AIX, Linux including Red Hat)

Platform virtualization (Citrix, Microsoft, VMware, Sun $\mathrm{xVM}, \mathrm{IBM})$

\section{KEY CHARACTERISTICS}

- Cost is greatly reduced and capital expenditure is converted to operational expenditure. This lowers barriers to entry, as infrastructure is typically provided by a third-party and does not need to be purchased for one-time or infrequent intensive computing tasks. Pricing on a utility computing basis is fine-grained with usagebased options and minimal or no IT skills are required for implementation.

- Device and location independence enable users to access systems using a web browser regardless of their location or what device they are using, e.g., PC, mobile. As infrastructure is off-site (typically provided by a third-party) and accessed via the Internet the users can connect from anywhere.

- Multi-tenancy enables sharing of resources and costs among a large pool of users, allowing for:

- Centralization of infrastructure in areas with lower costs (such as real estate, electricity, etc.)

- Peak-load capacity increases (users need not engineer for highest possible load-levels)

- Utilization and efficiency improvements for systems that are often only $10-20 \%$ utilized.

- Reliability improves through the use of multiple redundant sites, which makes it suitable for business continuity and disaster recovery. Nonetheless, most major cloud computing services have suffered outages and IT and business managers are able to do little when they are affected.

- Scalability via dynamic ("on-demand") provisioning of resources on a fine-grained, selfservice basis near real-time, without users having to engineer for peak loads. Performance is monitored and consistent and loosely-coupled architectures are constructed using web services as the system interface.

- Security typically improves due to centralization of data, increased security-focused resources, etc., but raises concerns about loss of control over certain sensitive data. Security is often as good as or better than traditional systems, in part because providers are able to devote resources to solving security issues that many customers cannot afford. Providers typically log accesses, but accessing the audit logs themselves can be difficult or impossible.

- Sustainability comes about through improved resource utilization, more efficient systems, and carbon neutrality. Nonetheless, computers and associated infrastructure are major consumers of energy.

\section{RISK MITIGATION}

Corporations or end-users wishing to avoid not being able to access their data — or even losing it — should research vendors' policies on data security before using vendor services. One technology analyst and consulting firm, Gartner, lists seven security issues which one should discuss with a cloud-computing vendor:

Privileged user access-inquire about who has specialized access to data and about the hiring and management of such administrators.

Regulatory compliance-makes sure a vendor is willing to undergo external audits and/or security certifications.

Data locations - ask if a provider allows for any control over the location of data.

Data segregation - make sure that encryption is available at all stages and that these "encryption schemes were designed and tested by experienced professionals".

Recovery - find out what will happen to data in the case of a disaster; do they offer complete restoration and, if so, how long that would take.

Investigative Support-inquire whether a vendor has the ability to investigate any inappropriate or illegal activity. Long-term viability - ask what will happen to data if the company goes out of business; how will data be returned and in what format.

In practice, one can best determine data-recovery capabilities by experiment: asking to get back old data, seeing how long it takes, and verifying that the checksums match the original data. Determining data security is harder. A tactic not covered by Gartner is to encrypt the data yourself. If you encrypt the data using a trusted algorithm, then regardless of the service provider's security and encryption policies, the data will only be accessible with the decryption keys. This leads to a follow-on problem: managing private keys in a pay-ondemand computing infrastructure.

\section{CONCLUSION}


Cloud Computing is a vast topic and the above report does not give a high level introduction to it. It is certainly not possible in the limited space of a report to do justice to these technologies. What is in store for this technology in the near future? Well, Cloud Computing is leading the industry's endeavor to bank on this revolutionary technology.

Cloud Computing Brings Possibilities:

- Increases business responsiveness

- Accelerates creation of new services via rapid prototyping capabilities

- Reduces acquisition complexity via service oriented approach

- Uses IT resources efficiently via sharing and higher system utilization

- Reduces energy consumption

- Handles new and emerging workloads

- Scales to extreme workloads quickly and easily

- Simplifies IT management

- Platform for collaboration and innovation

- Cultivates skills for next generation workforce

Today, with such cloud-based interconnection seldom in evidence, cloud computing might be more accurately described as "sky computing," with many isolated clouds of services which IT customers must plug into individually. On the other hand, as virtualization and SOA permeate the enterprise, the idea of loosely coupled services running on an agile, scalable infrastructure should eventually make every enterprise a node in the cloud. It's a long-running trend with a far-out horizon. But among big metatrends, cloud computing is the hardest one to argue with in the long term.

Cloud Computing is a technology which took the software and business world by storm. The much deserved hype over it will continue for years to come.

\section{REFERENCES}

[1]. www.wikipedia.com

[2].www.infoworld.com/article/08/04/07/15FE-cloud-computingreality $1 . \mathrm{html}$

[3].

www.wiki.cloudcommunity.org/wiki/CloudComputing:Bill_of_Rights

[4]. www.davidchappell.com/CloudPlatforms--Chappell. PDF

[5]. www.amazon.com

[6]. www.thinkgos.com/cloud/index.html

[7]. www.salesforce.com

[8]. http://www.sciencedaily.com/releases/

[9]. Chip Computer Magazine, December 2008 - Feb 2009 Edition 